community. Retrospective comparison of patients in the 2 groups found that patients in Group 1 had earlier seizure onset, longer epilepsy duration, and more patients with symptomatic etiologies, epileptic encephalopathy, and cognitive delay $(\mathrm{p}<0.001)$. Group 1 patients required more referrals to other specialties, and more frequent treatment with polytherapy, epilepsy surgery, ketogenic diet, and vagus nerve stimulator $(\mathrm{p}<0.001)$. Adult neurologists $(\mathrm{n}=86)$ and pediatric neurologists $(\mathrm{n}=29)$ surveyed indicated that adult neurologists have lower levels of confidence in diagnosing and treating severe forms of childhood-onset epilepsies $(\mathrm{p}<0.001)$ and epilepsies associated with cognitive delay $(\mathrm{p}<0.001)$. (Borlot F, Tellez-Zenteno JF, Allen A, Ali A, Snead OC, Andrade DM. Epilepsy transition: Challenges of caring for adults with childhood-onset seizures. Epilepsia 2014 Oct;55(10):1659-66).

COMMENTARY. Patients from tertiary centers present more complex needs and require more resources than age-matched patients from the community, and adult neurologists feel less confident in diagnosing and treating adult patients transferred with some childhood-onset epilepsies. The researchers propose that adult epileptologists should receive training in childhood-onset epilepsies. Alternatively, pediatric epileptologists should continue to follow more complex childhood-onset epilepsies in the adult or pediatric epilepsy clinic, provided the patient is seen regularly by an internist to attend to general systemic needs.

The most challenging childhood-onset epilepsies for adult neurologists are the epileptic encephalopathies with intractable seizures and cognitive delay. The well-known syndromes include West, Dravet, and Lennox Gastaut. Patients with Lennox-Gastaut syndrome followed for $>10$ years have severe cognitive delay in $75-99 \%$ cases and refractory seizures in $92 \%$.

\title{
References.
}

1. Camfield PR, Camfield CS. Pediatr Neurol. 2014 Jul;51(1):17-23.

2. Camfield PR, et al. Epilepsia. 2014 Aug;55 Suppl 3:24-8.

\section{DIFFERENTIAL DIAGNOSIS OF LENNOX-GASTAUT SYNDROME}

Epileptologists from Children's Hospital, Boston, and UCLA, California, discuss approaches to the differential diagnosis of Lennox Gastaut syndrome (LGS) and identification of a possible underlying etiology. The classic diagnostic criteria for LGS consist of a triad of features: multiple seizure types, abnormal EEG, and cognitive impairment. Onset is commonly between 3 and 5 years of age, with slight male preponderance, and is sometimes preceded by West syndrome. Tonic seizures during sleep are the classic feature used for diagnosis but in fact LGS has multiple concurrent seizure types: tonic, atypical absence, atonic, and myoclonic jerks. Nonconvulsive status epilepticus, lasting days to weeks, occurs in $50 \%$ patients, and sudden tonic or atonic falls, or "drop attacks," occur with the same frequency. The abnormal EEG shows slow spike-wave complexes (known originally as petit mal variant) at $2-2.5 \mathrm{~Hz}$ during wakefulness, and paroxysmal fast rhythms $(10-20 \mathrm{~Hz})$ during REM sleep. The MRI is abnormal in two thirds or more of patients with LGS. The IQ deteriorates over time, with $99 \%$ having cognitive delay by adolescence. Nonconvulsive status epilepticus is the most 
significant risk factor for severe cognitive impairment. Behavioral problems such as hyperactivity, aggression, and autistic traits occur in $50 \%$ cases.

The epileptic syndromes listed in differential diagnosis of LGS are Doose (myoclonic astatic epilepsy), Dravet (severe infantile myoclonic epilepsy), West (infantile spasms), and pseudo Lennox (atypical benign partial epilepsy). If diagnosis is not readily apparent from clinical history and exam, EEG, MRI, cardiac and eye exams, further tests suggested include DNA microarray, SLC2A1 (glucose transporter defect), CLN2 (late infantile neuronal ceroid lipofuscinosis), and TSC 1,2 (tuberous sclerosis). Diagnosis is important in determining prognosis and in treatment. (Bourgeois BFD, Douglass LM, Sankar R. Lennox-Gastaut syndrome: A consensus approach to differential diagnosis. Epilepsia 2014 Sep;55 Suppl 4:4-9).

COMMENTARY. A Working Group of experts chaired by Drs John M Pellock of Virginia Commonwealth University, Richmond, VA, and Dr James W Wheless of Le Bonheur Children's Hospital, Memphis, TN, met in Chicago, June 2012, and discussed diagnostic criteria and the management of LGS [1]. Investigators from Boston University; Tennessee, and Cincinnati, list FDA approved treatment options for LGS, including felbamate, lamotrigine, topiramate, rufinamide, and clobazam, and several others used off-label [2]. Investigators from Boston University and Kennedy Krieger Institute, Baltimore, outline surgical options that include lesionectomy and lobar resection, corpus callosotomy (effective in control of drop attacks), and vagus nerve stimulation [3]. Investigators from Johns Hopkins University and UCLA report a 50\% response to treatment with the ketogenic diet and a similar response to vagus nerve stimulation [4]. The Director of Epilepsy Information Service at Wake Forest University, Winston-Salem, NC, lists resources for caregivers and families of patients with LGS. They include the LGS Foundation, Epilepsy Foundation, Child Neurology Foundation and CNS, Charlie Foundation, and TS Alliance [5].

\section{References.}

1. Pellock JM, Wheless JW. Epilepsia. 2014 Sep;55 Suppl 4:1-3.

2. Montouris GD, Wheless JW, Glauser TA. Epilepsia. 2014 Sep;55 Suppl 4:10-20.

3. Douglass LM, Salpekar J. Epilepsia. 2014 Sep;55 Suppl 4:21-8.

4. Kossoff EHW, Shields WD. Epilepsia. 2014 Sep;55 Suppl 4:29-33.

5. Gibson PA. Epilepsia. 2014 Sep;55 Suppl 4:34-36.

\section{NEUROCUTANEOUS SYNDROMES}

\section{TUBEROUS SCLEROSIS COMPLEX AND ARACHNOID CYSTS}

Investigators at the Massachusetts General Hospital, Boston, MA, and Universitat Autonoma de Barcelona, Spain, assessed the prevalence and characteristics of arachnoid cysts in a cohort of 220 patients with tuberous sclerosis complex (TSC). A review of brain MRIs found arachnoid cysts in $12(5.5 \%)$ TSC patients compared to $0.5 \%$ in the general population. Ten $(83.3 \%)$ were males. Four patients $(33.3 \%)$ had also autosomal dominant polycystic kidney disease (ADPKD) due to a contiguous deletion of the TSC2PKD1 genes. Three patients (25\%) had 2 or more arachnoid cysts, of whom 2 also had ADPKD. One patient with an arachnoid cyst did not have tubers, subependymal nodules 